\title{
Reflexiones sobre la precariedad de algunas instituciones \\ El caso de un Banco Central independiente
}

\author{
JUAN URRUTIA \\ Universidad Carlos III de Madrid
}

Este trabajo exhibe tres niveles de lectura. En el primero se pretende elaborar algunas ideas relativas a la fragilidad de aquellas instituciones en las que interviene algún agente que, al estar relacionado con el Estado, no puede comprometerse irrevocablemente. Se arguye que esas institucionos son, en general, inestables a no ser que estén enraizadas en convenciones sociales que se expresen en un lenguaje inequívoco. En un segundo nivel estas ideas se aplican al estudio de un Banco
Central Independiente cuya problemática es, a su vez, el hilo conductor del trabajo. A lo largo de la exposición se plantean ciertos interrogantes que configuran un tercer nivel de lectura en el que se pretende acotar algunos problemas, comunes a filósofos, economistas y otros científicos sociales, tales como la racionalidad, la relevancia social de la falibilidad del conocimiento, la posibilidad de la acción comunicativa, $o$ el contraste entre universalidad y diversidad.

\section{Introducción}

Para algunos, la denominada Economía Institucional, heredera de una rica tradición, es una seria alternativa a la Economía Neoclásica '; para muchos, especialmente los historiadores económicos, las instituciones son cruciales para el desarrollo económico y para la emergencia del intercambio ${ }^{2}$; para casi todos, la propiedad privada es una institución sin la que los procesos económicos, tal como los conocemos, no podrían existir y todos vivimos rodeados, en todos los ámbitos de nuestra vida, de instituciones que condicionan la información que recibimos o las acciones que llevamos a cabo. El presente ensayo pretende examinar, de manera limitada y sumamente embrionaria, el papel y la estabilidad

${ }^{1}$ Ver a este respecto el libro de G. M. Hodgson Economics and Institutions, Polity Press, 1988, especialmente la parte tercera. Ver también una especie de continuación natural de este trabajo constituida por Economics and Evolution. Bringing Life into Economics que, publicado en 1993, ha sido traducido al castellano como Economía y Evolución. Revitalizando la Economia, y publicado por el Colegio de Economistas de Madrid, Celeste Ediciones, 1995.

${ }^{2}$ De hecho son los historiadores económicos en la estela de Douglas North los que han llamado la atención de manera generalizada en la época actual sobre la importancia de las instituciones. Ver, entre muchas referencias que se podrían ofrecer, el trabajo de D. North en el Joumal of Economic Perspectives, 5, 1991, y que lleva por titulo precisamene «Institutions». 
de esas instituciones que, por estar relacionadas con el Estado, o con el Gobierno como depositario de la sobcranía de éste, podríamos llamar políticas o, a nuestros efectos, instituciones macroeconómicas, ya que forman parte del ejercicio de la política macroeconómica de dicho Gobierno. Lo crucial de estas instituciones es que la soberanía del Estado anula la capacidad de autoobligarse del Gobierno, ya que no hay autoridad superior que fuerce el cumplimiento de sus promesas o de sus declaraciones ${ }^{3}$. Por esta razón su estabilidad o su precariedad es un problema muy real cuyo estudio constituye el objetivo principal del presente ensayo. El hilo conductor de la exposición está conformado por la secuencia de problemas que plantea la independencia de un Banco Central ${ }^{4}$; de ahí su subtítulo. Además del objetivo principal y del hilo conductor, este ensayo pretende también elucubrar sobre algunos puzzles conceptuales que parecen propicios para el examen filosófico.

La estrategia expositiva comienza por presentar, en el epígrafe 2, la principal consecuencia a nuestros efectos de la inexistencia de la capacidad de autoobligarse del Estado o del Gobierno; a saber, la posibilidad de inconsistencia intertemporal en la Política Monetaria y el consiguiente sesgo inflacionario. En el epigrafe 3 se presenta el papel que juegan las instituciones, ilustrándolo mediante el análisis de los efectos que dos instituciones estilizadas tienen sobre el sesgo inflacionario. Asimismo se introduce la distinción entre instituciones micro y macroeconómicas. Como ejemplo típico de estas últimas, en el epígrafe 4 se examina el papel y los efectos de un Banco Central independiente. Casi todo el resto del trabajo se dedica a examinar la posible precariedad de cse Banco Central en un contexto de juegos repetidos. El epígrafe 5 muestra el sentido más o menos preciso en el que esa institución, y las instituciones de diseño en general, son frágiles. El epígrafe 7 muestra las condiciones en las que estas instituciones y, en particular un Banco Central independiente, pueden llegar a ser estables: tienen que enraizarse en las convenciones propias de una sociedad concreta y elaborar y manejar un lenguaje con el que comuniquen

${ }^{3}$ En lo que sigue hemos de entender que el Gobierno es un Agente que ejecuta el poder (de hacer Política Económica) en nombre del Estado (Principal). Entre ellos habría una relación de agencia, que aqui se toma como dada, de forma que cuando hablamos de las preferencias del Gobierno no nos referimos a sus verdaderas preferencias, que serian las relevantes a efectos del contrato de agencia, sino a las que se podrían inferir de ese contrato que reflejan las del Estado. En cuanto que el Gobierno pone en práctica fielmente el clausulado del contrato de agencia nos encontramos con que sus anuncios de Política Económica no son obligaciones irrevocables. Sobre este problema, es decir, sobre los efectos que tener en cuenta la relación de agencia podria tener sobre la Política Monetaria, ver el trabajo de H. Grossman «Inflation and Reputation with generic policy preferences", y mi comentario al mismo en The Current Macrocconomic Consensus, incluido como vol. 1 de Actual Economic Problems in Economic Science, Servicio Editorial de la Universidad del País Vasco, 1989.

* Como el problema concreto de la independencia de un Banco Central no es más que el hilo conductor de cste ensayo, no debe esperarse una bibliografia exhaustiva al respecto. Con independencia de alguna referencia específica que se ofrecerá más adelante, una excelente bibliografía básica puede encontrarse en el trabajo de A. Cukierman Central Banking Strategy, Credibility, and Independence. Theory and Evidence, The MIT Press, 1992. 
sus intenciones y en relación con el cual se juzgue su reputación. En estos dos epígrafes y en el 6 se presta atención a algunos temas de interés filosófico como el de las metapreferencias, el de la racionalidad y sus paradojas, el de la presunta ventaja social de la falibilidad del pensamiento y el de posibilidad de comunicación a través de la acción. En el último epígrafe se resumen las enseñanzas, se concluye, y se apuntan otras direcciones de análisis.

Antes de entrar en materia hay que hacer hincapié en el hecho de que este ensayo tiene un carácter muy limitado y embrionario. Abstrae de mucha literatura no económica, se limita a utilizar la herramienta de la teoría de los juegos que, aunque sin duda relevante, no es la única aplicable al análisis de las instituciones ${ }^{5} \mathrm{y}$, sin duda, pone de manifiesto la poca autoridad de su autor en materias no económicas. A pesar de esto último no parece descabellado esperar que pudiera constituir un buen punto de encuentro entre filósofos, economistas, y otros científicos sociales.

\section{Punto de partida. Inconsistencia intertemporal y el sesgo inflacionario de la politica monetaria}

El punto de partida, tanto para el estudio del porqué de la independencia de un Banco Central como para el análisis de las instituciones macroeconómicas que aquí se propone, está constituido por el sesgo inflacionario que un Gobierno, que está interesado tanto en la actividad económica como en la eliminación de la inflación, puede llegar a generar en su uso de la Política Monetaria precisamente por la falta de credibilidad de sus anuncios antiinflacionarios en un contexto en el que esos amuncios son inconsistentes en el tiempo.

Desdc hace veinticinco años los economistas disponen de un modelo formal ${ }^{6}$ para entender por qué, aunque a largo plazo la inflación no influye en el nivel de actividad, a corto plazo sí puede instrumentarse una reducción de

s El locus classicus de la aplicación de la teoría de los juegos al análisis de las instituciones es el trabajo de Andrew Schotter The Economic Theory of Social Tnstitutions, Cambridge University Press, 1981. Otro trabajo clásico para otros planteamientos más generales es el de Jon Elster The Cement of Society, Cambridge University Press, 1989. El presente trabajo pretende establecer un punto de conexión entre el «estilo» de una y otra de estas publicaciones acudicndo para ello a los juegos dinámicos en tiempo real. En lo que sigue la teoría de los juegos se usa de manera profusa, pero muy informal, de forma que el lector cuidadoso quizá eche en falta un poco más de rigor. Proporcionarlo hubiera alargado la exposición más allá de lo razonable. Además de a North, de entre los grandes economistas que no usan la teoria de los juegos hay que citar como un estudioso profundo de las instituciones a Hayek. Sin cmbargo, y porque su trabajo en este campo es muy disperso, no se le citará directamente. A pesar de cllo en el último cpigrafe volveremos a él para resaltar las coincidencias con algunas de las reconstrucciones de su pensamiento elaboradas por John Gray en Hayek on Liberty, 2, ed., Oxford: Basil Blackwell, 1986, especialmente las resaltadas por el mismo Gray en su trabajo "Hayek, the Scottish School, and Contemporany Economics», capitulo 4 de The Boundaries of Economics, editado por G. C. Winston y R. F. Teichgraebor III, Cambridge University Press, 1988.

- Aunque hay precedentes ilustres hay que destacar el trabajo de Robert Lucas «Expectations and the Neutrality of Money», Yournal of Economic Theory, 4, 1972. 
la inflación a costa de ralentizar la actividad y generar desempleo. En este contexto surgen hace más de quince años, y de manera casi simultánea, los trabajos de Calvo y de Kydland y Prescott ${ }^{7}$ que nos hacen ver que esa capacidad de disciplinar la inflación puede estar limitada, incluso a corto plazo, por la falta de credibilidad antiinflacionaria de un Gobierno que también, y sobre todo, desea avivar el nivel de actividad.

La mejor manera de entender este punto de partida es plantear un juego estratégico elemental entre cl Sindicato $S$ (jugador 1) y el Gobierno $G$ (jugador 2) ${ }^{b}$. El Sindicato controla los salarios nominales y el Gobierno el nivel de precios. Con relación a su propia variable controlada cada jugador posce dos estrategias: $=0+$, es decir, mantener igual o aumentar (en la misma proporción) el salario nominal y el nivel de precios respectivamente. Ambos jugadores son racionales en el sentido de preferir más o menos y supondremos que esa racionalidad es conocimiento común ${ }^{9}$. La interacción entre estos dos jugadores produce cuatro posibles situaciones, reflejadas en el gráfico 1. En la situación A cada jugador juega la estrategia $=y$, en consecuencia, la inflación es nula y el salario real corresponde al que genera el nivel de actividad propio de la tasa natural de desempleo. En la situación B el Sindicato mantiene el salario nominal constante y el Gobierno eleva el nivel de precios generando inflación y un aumento del empleo gracias a la disminución del salario real. En la situación $\mathrm{C}$ el Gobierno mantiene el nivel de precios sin crear inflación y el Sindicato eleva el salario nominal generando una disminución del empleo debido al alza del salario real. Finalmente en la situación $\mathrm{D}$ ambos jugadores juegan $+\mathrm{y}$ generan inflación sin modificar el salario real que sigue correspondiendo a la tasa natural de desempleo. Para terminar la descripción del juego estratégico que nos sirve como punto de partida tenemos que especificar las preferencias de cada jugador sobre las cuatro situaciones. El Gobierno establece el siguiente orden $B A D C$, y el Sindicato $(A \sim D) C B$ con indiferencia entre las dos primera situaciones. Estas preferencias sobre situaciones, que reflejan las que sobre la inflación y la actividad tienen el Gobierno y el Sindicato, están representadas por los pagos que configuran la matriz del gráfico 1. Esta matriz de pagos, que representa el juego en forma normal, conforma un dilema del prisionero para sólo uno de los jugadores, el Gobierno y, por tanto, no es simétrico. En este gráfico

${ }^{7}$ F. E. Kydland y E. C. Prescott, «Rules rather than Discretion: The inconsistency of optimal plans", Joumal of Political Economy, 85, 1977. G. Calvo, "On the time incosistency of optimal policy in a monetary econony», Econometrica, 46, 1978.

* Toda la parte formal de este epigrafe y de los dos siguientes se toma, casi literalmente, del trabajo de Rafael Repullo «Sobrc la independencia de los Bancos Centrales», Papeles de Economia, 57, 1993, y de mi comentario a dicho articulo que aparece en el mismo sitio.

'Algo, sea $X$, es conocimiento comin entre dos individuos si ambos saben que $X$ es el caso y cada uno de ellos sabe que el otro sabe que él sabe que el otro sabe... que él sabe que $\mathrm{X}$ es el caso. Para finalidades posteriores conviene definir el conocimiento mutuo de orden $N$ de $\mathrm{X}$ como el conocimiento existente cuando la anterior recursión finaliza en $\mathrm{N}$ etapas, $\mathrm{N}$ finito. 
GRÁFICO 1

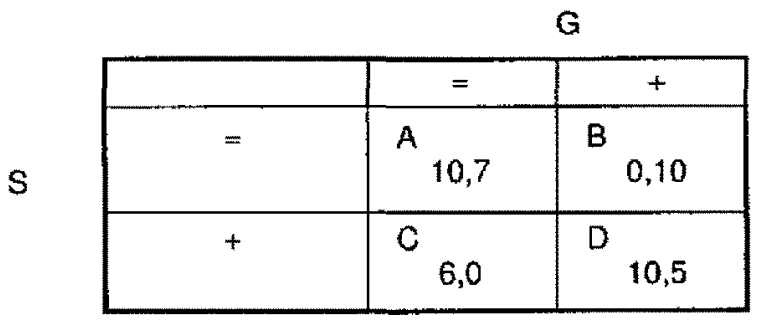

$y$, como es costumbre, la primera (segunda) entrada de cada casilla expresa el resultado para el primer (segundo) jugador. Como se ve, para el Gobierno el subir los precios es estrategia dominante ya que, haga lo que haga el Sindicato, a él siempre le viene mejor jugar + que $=$. Lo que más le gusta al Gobierno es la situación $\mathrm{B}$ que se podría obtener si se declara antiinflacionista $(=)$, el Sindicato le creyera (en cuyo caso jugaría también $=$ ) y, finalmente, el Gobierno jugara + engañando al Sindicato. Por otro lado, también es fácil de ver que el Sindicato no posee estrategia dominante y que quiere sobre todo no ser engañado, pero que si lo fuese preferiría mayor salario real aun a costa de una menor actividad y de un menor empleo.

Sabemos que la solución de un juego de estrategia, como es el descrito, tiene que ser tal que cada jugador esté haciendo lo mejor para él dado lo que hace el otro. Pues bien, ese equilibrio de Nash sólo se da en la casilla $D$ en donde el Gobierno infla la economía y el Sindicato eleva los salarios generando entre ambos una inflación positiva y una tasa de actividad que no es mayor, a pesar de los esfuerzos del Gobierno, que la correspondiente a la tasa natural de desempleo. Notemos que, aunque no se trata aquí de un dilema del prisionero propiamente dicho, tal como ocurre en ese juego, ambos jugadores preferirían estar en A pero acaban en $\mathrm{D}$. La clave de este resultado, denominado sesgo inflacionario, está en la falta de credibilidad del Gobierno por falta de capacidad técnica de autoobligarse. En efecto, como el Gobierno no puede obligarse a jugar $=$, todas sus declaraciones ${ }^{10}$ antiinflacionarias prometiendo jugar $=$ no son creíbles porque, tal como se ha dicho, si el Sindicato las creyera (y en consecuencia jugara $=$, no elevando los salarios nominales) el Gobierno jugaría + para disminuir el salario real y así aumentar el empleo y la actividad.

Ahora bien, para entender por qué se suele hablar en este contexto (y еп otros muchos) de inconsistencia intertemporal miremos al juego anterior en la forma extensiva que se reproduce en el siguiente gráfico 2:

19 Nótese que en esta forma nomal del juego no existe propiamente hablando la precedencia temporal implícita en el texto y que, además, contrariamente a lo que se afirma, no hay lenguaje en el que declarar lo que uno es. Lo primero se soluciona a continuación; pero la introducción formal de la conunicación entre agentes tiene que esperar al epigrafe 7. 


\section{GrŔFICO 2}

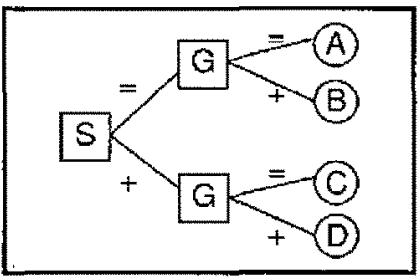

La solución de la forma extensiva funciona de atrás hacia delante. Miremos, pues, al Gobierno. Si está en la situación de arriba (cuando ya el Sindicato ha jugado $=$ ) jugará + , pues prefiere $\mathrm{B}$ a $\mathrm{A}$. Si está en la situación de abajo (cuando ya el Sindicato ha jugado + ) jugará + , pues prefiere $\mathrm{D}$ a $\mathrm{C}$. El Sindicato, sabiendo esto, jugará + , pues prefiere D a B. El resultado será, pues, aquí también $\mathrm{D}$ que, en este caso, es un equilibrio leader-follower con el Sindicato actuando como leader que puede elegir un punto en la función de reacción del Gobierno que actúa como follower. Ahora vemos con claridad que la estrategia $=$ no es consistente intertemporalmente para el Gobierno porque, cuando le toque jugar, nunca la elegirá, haga lo que haga cl Sindicato ${ }^{1}$.

\section{El papel de las instituciones}

Este resultado que acabo de exponer, y que se denomina sesgo inflacionario, permite introducir dos reflexiones que nos abrirán los ojos a la importancia y al papel de las instituciones en general y, en particular, a la problemática de las instituciones macroeconómicas.

Pensemos primero en la introducción de una legislación antiinflacionaria que prohíba que, en la negociación colectiva, se negocie una subida salarial acorde con la inflación pasada y exija que el Sindicato adapte la fijación de los salarios nominales a la evolución esperada del nivel de precios. Una legislación así, o un pacto del mismo tenor, constituiría una institución social cuyos efectos modifican drásticamente el equilibrio anterior y cuya introducción generaría reticencias obvias. En nuestro caso, éstas provendrían del Sindicato puesto que, formalmente, este pacto social deflacionario le arrebataría el liderato y le convertirá en un follower. Por su parte el Gobierno se convertiría en un leader que puede elegir un punto en la función de reacción del Sindicato. El resultado de la introducción de esta institución social se puede analizar

"Dependiendo de los contextos, esta inconsistencia intertemporal, para el Gobierno, de la estrategia = se manifiesta en que no corresponde a una estrategia que satisfaga el principio de optimalidad de Bellman o en que no puede constituir un equilibrio perfecto en subjuegos (Selten). 
en el contexto de la forma extensiva que acabamos de introducir y que sc representa, para el caso presente, en el siguiente gráfico 3:

\section{GRÁFICO 3}

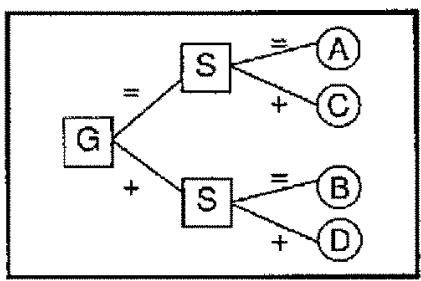

Como antes, la búsqueda del cquilibrio empieza por atrás, por el comportamiento del Sindicato. Si el Gobierno juega = el Sindicato elegiría jugar A, pues prefiere A a C. Si el Gobierno juega + el Sindicato clegiría D, pues prefiere D a B. Sabiendo esto el Gobierno elegirá =, pues prefiere A a D. El cquilibrio, por tanto, es ahora A y, como se observa, ha desaparecido todo sesgo inflacionario debido a que ya no hay inconsistencia temporal en el comportamiento del Gobierno.

Volviendo a la única forma normal consistente con las dos últimas formas extensivas, podemos, cn segundo lugar, explorar las consecuencias de sustituir un Sindicato como el modelado hasta ahora por un Sindicato que sólo tenga en cuenta el empleo y no preste atención al salario real. En términos realistas estaríamos hablando de sustituir un Sindicato de insiders que sólo vela por el salario real de los que ya tienen empleo y, por tanto, tienen una ventaja idiosincrásica sobre los que están en el paro, por un Sindicato de outsiders al que sólo interesa paliar la desventaja de los desempleados y conseguir colocarlos. Las preferencias de aquel Sindicato eran $A(\sim D) C B$ y las del de ahora son $B(A \sim D)$ C. Estas últimas, junto con las del Gobierno, que no cambian, conforman la siguiente matriz de pagos:

\section{GRÁFICO 4}

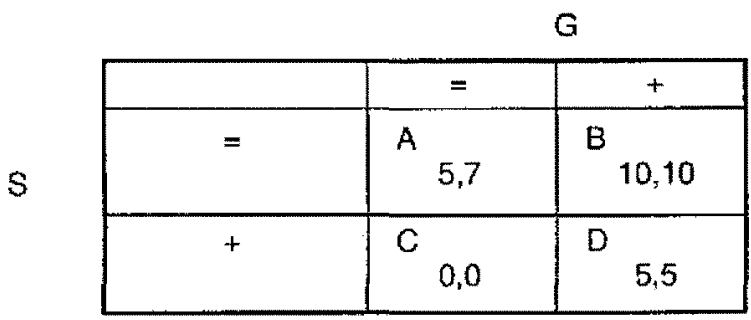


Para el Gobierno + sigue siendo estrategia dominante. Lo novedoso es que ahora la estrategia $=$ es dominante para el Sindicato. El resultado es pues $\mathrm{B}$, una solución en la que hay inflación y también un empleo superior al natural debido a la disminución de los salarios reales. Aquí también la sustitución de una institución por otra ha generado un cambio en el resultado. Estos dos ejemplos muestran la importancia que tienen las instituciones en la determinación del resultado de cualquier mecanismo económico a de cualquier proceso social. Las instituciones siempre funcionan modificando la información disponible y/o el conjunto de acciones o estados sobre los que hay que ejercitar la decisión, social o individual. A veces prohiben algunos de esos estados o acciones, a veces introducen uno nuevo; pero de una u otra forma suelen traer consigo un cambio dramático en el resultado de las decisiones individuales o colectivas.

Los ejemplos económicos de la importancia de las instituciones son muy numerosos. Demorémonos un instante en el campo microeconómico. El área general denominada de implementación, o también diseño de mecanismos, cubre casi con exactitud el problema del diseño de instituciones. Quizá el ejemplo más conocido de esta literatura sea el del diseño de mecanismos de revelación sincera de preferencias que permitan la implementación del óptimo de Lindahl en una economía con bienes públicos. El incentivo a mentir que tiene todo aspirante a polizón puede ser alineado en la dirección adecuada introduciendo mecanismos que hacen que cada individuo enfrente las mismas consecuencias que enfrentaría en condiciones de competencia en un mercado para un bien público personalizado o, en otras palabras, mecanismos que confrontan a cada agente, no con su problema individual, sino con el problema social ${ }^{12}$. En el área de la elección social encontramos ejemplos claros del dramatismo de los cambios en el resultado producidos por pequeños cambios en los objetos de la elección social (por ejemplo, candidatos en una votación) o por cambios en los procedimientos de votación ${ }^{13}$. En cl área del estudio

${ }^{12}$ La referencia obligada es el trabajo de T. Groves y J. Ledyard "Optimal Allocation of Public goods: A solution to the free-rider problem», Econometrica, 4, 1977.

1. Ver el capítulo 9 «Majority Voting and Scoring Methods" de la Monografía núm. 15 de la Econonetric Society Monographs de H. Mouin, Cambridge University Press. En él se explica, de una forma simple y clara, cómo los intentos de generalizar el procedimiento de la mayoría a la elección entre más de dos candidatos desembocan inmediatamente en problemas que resaltan la primera parte de lo indicado en el texto: cambios en los procedimientos (de Borda a Condorcet, o viceversa) cambian dramáticamente el resultado. Por otro lado sabemos que la eliminación de un candidato (o de una alternativa cualquiera de elección), puede resultar, para ciertos perfiles de preferencias, en cambios bruscos en el resultado de diversos tipos de elección. La exigencia de que esto no ocurra cuando la opción eliminada es irrelevante (en el sentido de que no sería elegida) se denomina Independencia de Alternativas Irrelevantes (IAI) y constituye una pieza clave del famoso Teorema de Imposibilidad de Arrow. 
de las propiedades de las subastas nos encontramos con que el resultado de una subasta depende mucho de su diseño ${ }^{14}$.

Estos ejemplos generales, entresacados de la microeconomía, hacen reflexionar sobre tres puntos que resultan importantes para lo que sigue. Primero, el análisis puede efectuarse de una forma cuasi-ingenieril porque los resultados que surgen del funcionamiento de esos mecanismos pueden imponerse por la autoridad del Estado incluso si el mecanismo de que se tratc está mal diseñado o es difícil de entender (como ocurrió, por ejemplo, en la subasta de frecuencias de radio en Nueva Zelanda) o, simplemente, no le gusta a alguien. Segundo, los cambios que la introducción de estas instituciones microeconómicas traen consigo no ponen en juego, en general, las costumbres o la cultura de la sociedad en cuyo seno se las hace funcionar. En algún caso específico como, por ejemplo, el cambio de un sistema electoral desde uno proporcional a otro mayoritario, no podría decirse lo mismo; pero notemos que ese tipo de cambios se hacen precisamente para ponerse en línea con la cultura prevalente. Tercero, es muy probable que la puesta en marcha de estas instituciones microeconómicas plantee problemas de comunicación entre agentes, ya que el mecanismo introducido puede exigir la transmisión de mensajes complicados para los que hace falta una cierta creación de lenguaje.

Hechas cstas precisiones, volvamos al tema genérico del diseño de instituciones, ahora en el campo macroeconómico. Tal como ponen de manifiesto los dos ejemplos estudiados en esta sección, el diseño de instituciones se complica en cuanto pasamos al campo de la política macroeconómica. En un caso, el de la legislación antiinflacionaria o pacto equivalente, uno de los jugadores es el Estado, el único, tal como ya se ha indicado, que no puede ser obligado por nadie a cumplir su compromiso o sus amenazas. El otro caso, el del Sindicato de outsiders, pone de manifiesto que si se opera con un Sindicato u otro no es algo que se pueda elegir en un momento dado; sino que parece depender más bien de las costumbres o de la cultura de un país determinado. Ambos ejemplos plantean cambios institucionales lo suficientemente radicales como para pensar que habría que desarrollar un lenguaje en cuyo contexto pueda hablarse de credibilidad con propiedad. Son este tipo de problemas los que se ponen en juego en cuanto confrontamos cualquier problema institucional de tipo macroeconómico. Pensemos en la Unión Monetaria, en los criterios de Maastricht, en el Pacto de Estabilidad o en el Euro. Los avatares, bien conocidos, de estas nuevas instituciones de política macroeconómica demues-

${ }^{14}$ La literatura sobre subastas muestra también que cambios en su diseño, es decir, lo que aquí llamariamos modificaciones institucionales, pueden traer consigo cambios importantes en los resultados relativos al ingreso potencial obtenido por quien es propietario del objeto subastado y a la eficacia en la asignación del objeto a la persona que más lo aprecia. La aportación pionera en este campo es la de $W$. Vickery contenida en kCounterspeculation, Auctions and Competitive Sealed-tenders», Joumal of Finance 1967. Una introducción a la literatura sobre subastas más reciente y muy útil es la de P. Milgrom en «Auction and Bidding: a Primer», Journal of Economic Perspectives, 1989. 
tran que los cambios institucionales no son fáciles de imponer a contrapelo de las costumbres y la cultura de cada país y que, para ser exitosas y enraizarse, estas instituciones necesitan a veces la creación de lenguaje.

Quizá el ejemplo de institución macroeconómica mejor estudiado sea el de un Banco Central y su independencia. Su examen puede darnos pistas respecto a los problemas planteados.

\section{Un Banco Central independiente}

Volvamos al punto de partida en el que se manifestaba la inconsistencia intertemporal de la política antiinflacionaria del Gobierno. Mientras éste pueda generar inflación y mientras sus preferencias muestren un deseo de incrementar la actividad económica mediante la disminución de los salarios reales, cualquier declaración antiinflacionaria que hiciera, suponiendo que pudiera hacerla y que fuera entendida, no sería creída por el Sindicato.

Ante esta situación cabría una aplicación ingenua de lo aprendido hasta ahora sobre instituciones y que sería muy propia de la ingeniería social: prohibamos al gobicrno jugar la estrategia +. Esta propuesta es ingenua porque, como ya ha sido recordado en varias ocasiones, el Gobierno (o mejor el Estado de quien deriva su autoridad) no puede ser obligado a no jugar la estrategia + . Pero su examen es ilustrativo. Si la prohibición se efectuara, al Gobierno sólo le quedaría la estrategia $=\mathrm{y}$, en estas condiciones, el Sindicato también elegirá, dadas sus preferencias, la estrategia $=$. En consecuencia podríamos decir que la situación $A$, en la que ambos jugadores juegan la estrategia $=$, es, de manera trivial, un equilibrio en estrategias dominantes (y por ende un equilibrio de Nash) en el que la economía está sobre la tasa natural de desempleo y en el que no hay sesgo inflacionario. Esta propuesta prohibicionista puede parecer no sólo ingenua, sino también estúpidamente voluntarista, pero es la que yace en el fondo de la sugerencia de llevar a la Constitución de los países una regla sobre el incremento de la oferta monetaria y la que late en la discusión sobre las ventajas de las reglas sobre la discreción a la hora de llevar a cabo la Política Monetaria. Sin embargo ni esa sugerencia ni la implantación de una regla han sido llevadas a la práctica en casi ningún lado. La razón seguramente es triple. Inflar la economía tiene efectos reales procurando una tasa de desempleo por debajo de la natural al menos a corto plazo, tal como nos hace ver el citado resultado de Lucas. Nadie está dispuesto a renunciar del todo a hacer uso de esa posibilidad ante shocks depresivos. Y, además, es posible *aparcar» el uso de la estrategia inflacionaria sin necesidad de renunciar a ella para siempre.

Para ilustrar esta constelación de ideas no hay nada mejor que examinar el argumento más corriente en favor de la independencia de un Banco Central. El Gobierno, a la vista del sesgo inflacionario, y ante la imposibilidad de engañar 
al Sindicato respecto a sus verdaderas preferencias, delega la ejecución de la Política Monetaria (y, en consecuencia, la determinación del nivel de inflación) en un Banco Central con preferencias más antiinflacionarias que el Gobierno (aunque no necesariamente indiferente al nivel de actividad) y con la independencia o autonomía necesaria para no tener que sucumbir a las presiones que el propio Gobierno tratará de ejercer. Digamos que las preferencias del Banco Central son tales que los posibles resultados del juego con un Sindicato de insiders que conserva las suyas son ordenadas, tal como refleja la matriz de pagos representada en el siguiente gráfico 5, como $\mathrm{ACBD}$ :

GRÁFICO 5

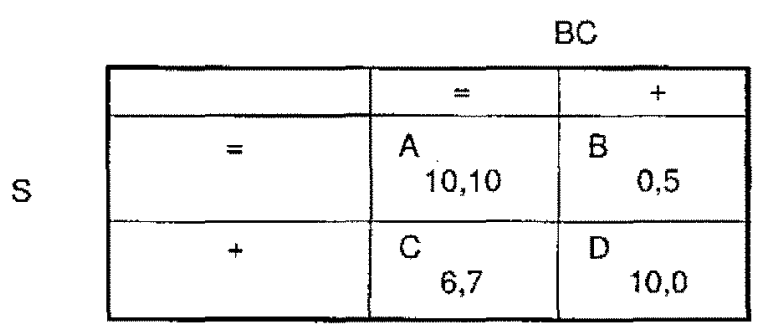

Como se observa con toda facilidad, no subir los precios es ahora estrategia dominante para el Banco Central y el conocimiento comín de esto hace que el nuevo equilibrio de Nash corresponda ahora a la casilla A. En esa situación no hay inflación y la economía se encuentra sobre la tasa natural de desempleo. La delegación ha funcionado como un sustituto de la capacidad de autoobligarse y el riesgo inflacionario ha sido eliminado.

La delegación (o, como decíamos antes, el aparcamiento) de la Política Monetaria en un Banco Central ticne los mismos efectos que la propuesta prohibicionista ingenua con que se abrió este epígrafe o que la posible legislación antiinflacionaria antes mencionada. Sin embargo, a diferencia de estos ejemplos, utilizados aquí de manera retórica, esta idea de la autonomía o independencia de un Banco Central, aunque parezca retórica, ha sido de las más fructíferas en los últimos quince años, tanto en su aspecto teórico como en la mediación de los resultados de su implantación y tal como pone de manifiesto el ya citado libro de Cukierman.

Su desarrollo teórico ha ocupado varios frentes. Debería ser obvio que el deseo de avivar la actividad económica no es el único que puede estar en la base del sesgo inflacionario, sino que también pueden contribuir a él el deseo de incrementar los ingresos fiscales a través del «seignoriage», el intento de mejorar la balanza de pagos a través de la devaluación o la aspiración a proporcionar una estabilidad financiera. Nada de esto nos ocupará aquí, pero también debería estar claro que el análisis puede, y debe, complicarse 
en diferentes direcciones algunas de las cuales sí serán de interés en lo que sigue. En efecto, el análisis, para ser exhaustivo, debe efectuarse en el contexto de juegos repetidos y complicarse con la consideración de diversos escenarios diferenciados según las posibilidades de asimetría informacional. Asimismo, pueden añadirse diversas consideraciones enriquecedoras. El Banco Central puede estar sometido a un contrato incentivador, sin necesidal de confiar en las notorias preferencias antiinflacionarias de un Gobernador (o similar). Es posible también que esté condicionado por la estructura política de sus órganos de gobierno y siempre es de interés confrontarlo no sólo al Sindicato sino también a otras ramas del ejecutivo, por ejemplo al Ministerio de Hacienda.

El trabajo empírico también ha sido profuso. Ha proporcionado tanto mediciones diversas de la independencia (desde la legal hasta la financiera) como correlaciones cntre esas medidas y la inflación (o su volatilidad) o la actividad cconómica. Asimismo ha contribuido a la exploración de los determinantes de dicha independencia. En cste punto es muy importante examinar la evidencia empírica, pues pudiera ser que ésta fuera suficiente como para zanjar de antemano, por irrelevante e intempestivo, el intento de este ensayo de sugerir la posible fragilidad de esas instituciones que, como ésta de un Banco Central independiente, pueden considerarse de diseño en el sentido de que son introducidas en cualquier economía en razón de sus propiedades teóricas y sin consideración alguna de las costumbres o de la cultura de cada país. Sin embargo no parece que la evidencia empírica disponible hoy, dada su naturaleza, pueda llegar a zanjar nada. Se trata, en efecto, del examen de un panel de países, cada uno con un Banco Central al que se ha dotado de mayor o menor independencia en fechas diversas. Parece observarse que, cuanto más independientc es el Banco Central de un país, menor es su tasa media de inflación en los últimos años y también que la tasa de crecimiento medio durante esos años no tiene relación significativa alguna con la independencia del Banco Central. Sin embargo bien pudiera ocurrir que estas correlaciones fueran espúreas y recogieran otros factores comunes que expliquen simultáncamente la caída de la inflación y la independencia del Banco Central. Para desacreditar de antemano el esfuerzo de alertar sobre la fragilidad de instituciones de diseño sería necesario disponer de cvidencia que justificara la creencia de que los costes (en términos de desempleo, por ejemplo) incurridos por la concesión de autonomía a un Banco Central son independientes de la historia, la costumbre o las tradiciones de cada país. Como a mi leal saber y entender esta evidencia no existe todavía, merece la pena seguir explorando la presunta fragilidad de las instituciones de diseño. 


\section{Fragilidad de las instituciones de diseño y algunos puzzles intelectuales}

A los razonamientos de la sección anterior cabe oponer dos objeciones que no son triviales y que conforman un puzzle intelectual interesante en sí mismo. En efecto, es difícil de entender cómo es posible que el Gobierno no esté contento con sus propias preferencias y que prefiera tener las del Banco Central. ¿Qué significa que un agente económico no aprecie sus preferencias cuando la única fuente de apreciación son sus preferencias? Para un economista la manera más natural de entender este puzzle es suponer que, además de preferencias definidas sobre el espacio de elección (inflaciốn y desempleo en nuestros ejemplos), hay una especie de metapreferencias sobre el espacio de preferencias.

Formalmente deberíamos estar satisfechos con esta explicación. De hecho, el principal interés del famoso artículo de A. Sen sobre Rational Fools radica precisamente en mostrar que el supuesto de existencia de preferencias de segundo nivel o metapreferencias permite explicar algunos comportamientos contrarios al egoísmo de una manera formalmente análoga a la empleada cuando trabajamos con sólo preferencias de primer nivel que se supone representan ese egoísmo. En efecto, decisiones o comportamientos observados en el campo moral (o en cualquier otro campo, como el de la adicción, en el que se ponga en juego la debilidad de la carne frente a la diligencia del espíritu) que parecen ser estrictamente dominados de acuerdo con las preferencias de primer nivel, son internamente consistentes en cuanto suponemos la existencia de un cierto tipo de preferencias de segundo nivel. Sin embargo esta solución formal no es del todo satisfactoria, pues deja sin explicar de dónde surgen estas preferencias de segundo nivel. En un sentido esa pregunta es tan ilegítima como preocuparse por el origen de las preferencias de primer nivel (por algún sitio hay que empezar); pero en otro sentido es tan legítima como preocuparse por la formación o modificación endógena de las preferencias primarias. Por tanto parece que podríamos no estar del todo satisfechos con la propuesta de Sen. Una manera de eliminar esta insatisfacción sería por ejemplo, y utilizando un lenguaje freudiano, suponer que preferencias y metapreferencias pertenecen respectivamente al ego y al superego, quienes se enfrentan en un juego de estrategia. Podríamos decir que el ego es el Gobierno con sus preferencias primarias y que el superego actúa de tal manera que el comportamiento del Gobierno acaba siendo como el del Banco Central. Las preferencias que atribuimos a éste serán pues la composición de las preferencias del Gobierno con las del superego.

Una vez más la solución parece meramente formal, pues no deberíamos estar satisfechos hasta que supiéramos cómo se alcanza un equilibrio en el juego entre el ego y el supcrego poseedores de unas y otras preferncias. 6 Vencerá el superego detentador de las metapreferencias, como en la historia de Ulises y las Sirenas, mediante el expediente de atar el ego al mástil? Quizá; pero 
no cabe desestimar la posibilidad de que ocurra lo del falso cuento popular de la rana y el escorpión. Éste convence a aquélla de que le atraviese el río utilizando el argumento de que no le clavará su aguijón pues perecerían ambos. Sin embargo a mitad del río el ego del escorpión sigue la llamada de su naturaleza (sus preferncias primarias) desestimando no sólo su superego, sino también su instinto de supervivencia, y ambos perecen ahogados ${ }^{15}$.

Este último ejemplo nos lleva a la segunda objeción. No hay, en efecto, ninguna garantía supcrior de que el Gobierno no derogue la delegación genćrica otorgada al Banco Central o no denuncie el contrato incentivador que ha firmado con el banquero central. Es pues evidente que un Banco Central, aunque sea formalmente independiente, es una institución frágil en el sentido elemental de que su permanencia depende de la voluntad de un jugador que no pucde ser disciplinado y que posee unas preferencias básicas para las que mantener su independencia no es la mejor opción. Es también frágil en otros sentidos más profundos, pero para estudiarlos necesitamos movernos en un escenario dinámico de juegos repetidos en los que en cada momento de tiempo se juega el juego estático, bien en forma nomal, bien en forma extensiva. En este nuevo escenario las estrategias son secuencias de $=\mathrm{y}$ de $+\mathrm{y}$ hay que distinguir con cuidado si el tiempo es virtual o real, y si la información es completa o incompleta o constituye o no conocimiento común.

En lo que queda de esta sección nos concentraremos en modelos en los que el tiempo es virtual. Es decir, la repetición es virtual y las estrategias son secuencias de estrategias puntuales establecidas desde el principio y de una vez por todas de forma que no hay lugar para el aprendizaje ni para redefinir la estrategia.

Como primer caso examinemos lo que ocurre con información completa. Aparte posibilidades con horizonte infinito o con horizonte finito y alguna impaciencia, que resultan muy artificiosas en el contexto presente y exigirían la introducción de estrategias condicionales, la información completa fuerza que la solución sea la mera repetición de la solución estática. Si es el Gobierno el que juega observaremos en cada período el sesgo inflacionario, pues es la única solución que es self-enforcing (autovinculante) para este agente. Similarmente si es el Banco Central el que juega observaremos en cada período ausencia de sesgo inflacionario y tasa natural de desempleo. Podríamos decir que, en este contexto, el Banco Central independiente es necesario, pero es

13. Ver «Rational Fools: A Critique of the Behavioral Foundations of Economic Theory", Philosophy and Public Affairs, 6, 1977. Ver también Egonomía de J. Elster, Gedisa, 1977. Merece la pena indicar aquí que el juego entre el ego y el superego puede ser estudiado como va a serlo el juego entre el Banco Central y el Sindicato en los proximos epigrafes: de forma dinámica y en tiempo real, Queda para el lector aplicar las próximas ideas a este juego freudiano y extraer las implicaciones. En cualquier caso caben también explicaciones que no caen dentro de la idea de metapreferencias ni tampoco apelan a la teoría de los juegos, sino que se centran en la formación endógena de preferencias. Ver al respecto el trabajo de J. C. García-Bermejo «Racionalización intemporal con cambio de prefcrencias", Investigaciones Económicas, mayo-agosto 1983. 
tan frágil como en el caso estático y por las mismas razones, ya que la introducción del tiempo no ha cambiado nada.

Pensemos ahora en el caso de que la información sea incompleta. En este contexto se puede mostrar fácilmente que, incluso con horizonte finito, hay condiciones que hacen de la reputación un sustituto de la capacidad de compromiso. Pensemos que el Sindicato no sabc si está confrontando un Gobierno débil (con preferencias como las que hemos atribuido aquí al Gobierno) o un Gobierno fuerte (con preferencias como las atribuidas aquí al Banco Central). En estas circunstancias se puede mostrar que hay un equilibrio secuencial ${ }^{16}$ que sostiene la solución $\mathrm{A}$ en todos los períodos del juego y en el que al Gobierno se le asigna una probabilidad alta de ser fuerte (su reputación). Podríamos decir que ahora el Banco Central independiente ya no es necesario, pero también podemos interpretar el resultado como la solución que se obtendría cuando el jugador es un Banco Central independiente cuyas preferencias no se conocen y cuya reputación tiene que ser elaborada en el juego ${ }^{17}$.

Bajo esta interpretación la reflexión genérica de los expertos en teoría de juegos, que no sus construcciones formales, nos hace ver que la reputación es algo frágil y que es tanto más poderosa, como sustituto de la capacidad de compromiso, cuanto más frágil es ${ }^{18}$. Si su reputación es frágil y la institución sirve en cuanto adquiere reputación, podemos concluir, sin abusar del lenguaje, que la institución es frágil.

La institución de un Banco Central, y por muy independiente que éste sea, también es frágil en el sentido de que puede ser peligrosa. El análisis de este peligro corresponde más bien a un ejercicio intelectual distinto. Supongamos que, a diferencia del ejercicio del párrafo anterior, la economía se encuentra en un equilibrio del juego repetido en el que (quizá porque se conocen muy bien las preferencias del Gobierno) en cada periodo de tiempo se está jugando por parte de ambos jugadores la estrategia +. El Gobierno decide introducir la independencia del Banco Central: un cambio de régimen. Como

${ }^{10}$ Esta noción de equilibrio es muy parecida a la de equilibrio perfecto en subjuegos aunque más fácil de cómputo, pues hace uso del teorema de Bayes al actualizar la probabilidad asignada al evento de que el Gobierno sea fuerte. Esa noción de equilibrio para juegos en forma extensiva repetidos en tiempo vritual fue introducida por D. M. Kreps y R. Wilson en «Sequential Equilibria», Econometrica, 50, 1982.

"Ver D. M. Kreps y R. Wilson, «Reputation and Imperfect Information», Joumal of Economic Theory, 27, 1982.

14 Kreps y Wilson en el trabajo referido en la nota anterior dicen literalmente: «tenemos un modelo en el que la reputación es fácilmente eliminada..., el poder de la reputación parece estar positivamente relacionado con su fragilidad». Intuitivamente esto último es fácil de aceptar. Cuanto menos plausible es un comportamiento observado, en el sentido de que se desvía mucho del comportamiento que se entiende por "normal», más creemos que el comportamiento observado pertenece a un agente sanormab. En cuanto a la facilidad para eliminar la reputación no se refiere tanto al hecho de que pequeños cambios o errores destruyan el equilibrio secuencial con reputación; sino a que, en un mismo contexto, la "emergencia» del equilibrio secuencial con reputación puede no darse para un cambio infinitesimal de la reputación inicial. 
quizás la reputación del Banco Central está por establecer, es posible que su adquisición genere muchos períodos de desempleo. En efecto, este nuevo Banco Central está obligado a jugar = muchas veces, juegue lo que juegue el Sindicato. Y si, para testar la fortaleza de este Banco Central, el Sindicato juega + , pueden darse, como decíamos, muchos períodos de desempleo, ya que si el Banco Central pasa a jugar + se delataría como débil haciendo imposible alcanzar la solución $\mathrm{A}^{19}$.

Este experimento intelectual de cambio de régimen es, sin embargo, algo extraño en el contexto de tiempo virtual. En este contexto, en efecto, sólo cabría, en puridad de principios, comparar equilibrios alternativos con una u otra institución y asi lo hemos hecho en este epígrafe. La idea de cambio de régimen nos remite a un contexto de tiempo real más acogedor para esta idea. A este contexto dedicaremos el epígrafe 7, pero antes merece la pena explorar las consecuencias de reconocer que la racionalidad puede no ser conocimiento común.

\section{La potencialidad de la ignorancia de la racionalidad}

En el contexto de información completa hemos visto cómo, aparte de posibilidades que calificábamos de artificiosas, la solución corresponde a la situación $D$ del juego estático. Sin embargo en tiempo finito, o incluso en el juego estático, se puede obtener la solución A siempre que ensanchemos el espacio de estrategias y permitamos algo de ignorancia.

Pensemos que el espacio de estrategias contiene, además del par $(=,+)$ estrategias condicionales del tipo wharé $\mathrm{X}$ si tú haces $\mathrm{Y}$ », en donde $\mathrm{X}, \mathrm{Y}$ $\in(=,+)$. Es cierto que, además de ensanchar el espacio de estrategias, la admisión de este tipo de cstratcgias condicionadas parece admitir una posibilidad de comunicación que no está modelada. Nos ocuparemos de esto más adelantc; pero ahora se trata de ver cómo este tipo de estrategias puede permitir alcanzar la situación A en la que, como se recordará, Gobierno y Sindicato mantienen los precios y los salarios nominales respectivamente.

Consideremos en concreto la estrategia tit-for-tat que comienza jugando = y luego jugando $=$ si y sólo si el otro ha jugado $=$. Pues bien, siempre que haya un cierto grado de ignorancia sobre la matriz de pagos puede mostrarse que, incluso con horizonte finito, la situación A se obtendrá en un buen número de períodos formando parte de un equilibrio secuencial ${ }^{20}$.

Hay, sin embargo, otra forma alternativa de introducir un cierto grado de ignorancia. Se trataría de romper la hipótesis de conocimiento común sobre

\footnotetext{
${ }^{19}$ Ver el trabajo de D. Backus y J. Drifill «Rational Expectations and Policy Credibility Following a Change in Regimen, Review of Economic Studies, 52, 1985.

20 Ver «Rational Cooperation in the Finitely Repeated Prisioner's Dilemma», de D. M. Kreps, P. Milgrom, J. Roberts y R. Wilson, Joumal of Economic Theory, 27, 1982.
} 
la propia racionalidad ${ }^{21}$. No es que haya incertidumbre sobre la racionalidad ajena; sino que lo que hay es un mero conocimiento común de orden $\mathrm{N}, \mathrm{N}$ finito, de esa racionalidad ajena. El examen de esta posibilidad detectada por Auman no aporta ninguna novedad al análisis de la fragilidad de las instituciones de diseño; pero tiene interés en sí misma y por los corolarios que de ella pueden extraerse.

Enfrentémonos pues al juego de información completa del gráfico 1 de nuevo y consideremos la siguiente estrategia condicionada de cualquiera de los dos jugadores, una versión de la estrategia tit-for-tat: «No subiré los salarios (precios) si no subes los precios (salarios); pero en cuanto esto ocurra subiré los salarios (precios) en todos los períodos». Si la racionalidad de cada agente es conocimiento común, esta estrategia condicionada no es creíble. Sin embargo, si la racionalidad de cada agente es sólo conocimiento mutuo de orden 1 surge la siguiente posibilidad. Aunque el Sindicato sepa que, de hecho, el Gobierno quiere subir los precios, es posible que sospeche que el Gobierno no sepa que él lo sabe y que, en consecuencia, el Gobierno pretenda aprovecharse de esa presunta ignorancia sindical disfrazándose de antiinflacionario (jugando $=$ ) en cuyo caso al Sindicato le vendrá bien no subir los salarios (jugar =).

Auman muestra que el razonamiento continúa valiendo en presencia de conocimiento mutuo de orden $2 \mathrm{y}$, aunque no lo prueba, sospecha que «se puede llevar el conocimiento mutuo (de la racionalidad) a cualquier orden finito, más acá del conocimiento común, y todavía obtener el mismo resultado, que los jugadores estarán motivados a jugar estrategias mutuamente beneficiosas pero aparentemente irracionales».

De este tipo de razonamiento pueden intentar extraerse dos corolarios, uno relativo a la Racionalidad con mayúscula, otro relativo a la falibilidad del conocimiento y a la sociedad abierta. Respecto a la Racionalidad sólo cabe aquí motivar la reflexión mediante algunas preguntas retóricas que conectan con el problema de los «Rational Fools», ya examinado en el epigrafe 5, aunque no en el sentido de tachar de locos a los que son meramente racionales, sino en el sentido de pretender ensanchar el concepto de racionalidad hasta abarcar conductas que, sin metapreferencias, muestran un cierto desprecio del egoísmo. ¿Llamaríamos irracional a la estrategia $=$ si sólo forma parte de la exploración de las propias preferencias? Si este fuera el caso, ino es racional sospechar que el otro pueda ser algo irracional en relación con toda la matriz?; ¿no es racional aprovecharse de la posible irracionalidad ajena? Y, finalmente,

${ }^{2}$ La importancia de pasar de conocimiento común a conocimiento mutuo de orden $\mathrm{N}$ (ver nota 9) fue puesta de manifiesto por P. Milgrom y J. Roberts en aPredation, reputacion and entry deterrence», Joumal of Economic Theory, 27, 1982, en relación a la incertidumbre sobre la matriz de pagos, o sobre esta misma incertidumbre, $y$ asi es recogida en el trabajo referenciado en la nota anterior. En el texto se sigue la sugerencia de Auman de aplicar las ideas de Milgrom y Roberts no a la incertidumbre sino a la propia racionalidad. Ver el manuscrito do Auman «Integrating Irrationatity into Game Theory", redactado en 1988 y, que yo sepa, no publicado. 
ino es racional aprovecharse del disfraz de irracional del otro jugador y disfrazarse de irracional? ${ }^{22}$

Con relación a la falibilidad del conocimiento surge una reflexión de otro cariz. Cuando lo que está en juego es el conocimiento por parte de un agente de un hecho de la naturaleza, la falibilidad del conocimiento puede expresarse mediante la probabilidad positiva de error. En este caso la sociedad abierta puede considerarse como con un «aparato epistémico» ${ }^{23}$ que de la actividad libre de cada uno, deduce y agrega una revaluación de la probabilidad de error, de forma que éste sea cada vez menor. Esta conclusión optimista puede ponerse en duda si ahora concebimos el conocimiento como conocimiento mutuo de otro agente (de su racionalidad en el ejemplo, o de su solidaridad o de cualquier otra característica). En este caso la falibilidad del conocimiento puede entenderse de otra manera. Sigue cabiendo que el conocimiento tenga una probabilidad de error pero ahora surge la posibilidad adicional de que un agente, aun calibrando bien esa posibilidad de error, actúe como si la calibrase mal. Y surge porque al creer que el otro no sabe que él calibra bien, espera del otro que actúe de una forma tal que le interese a él mismo actuar como si calibrase mal. Si esta posibilidad se hace efectiva, el presunto «aparato epistémico" constituido por la sociedad abierta exhibiría un cierto ruido que debilita el anterior argumento a su favor. La actividad libre de cada uno, basada ahora no sólo en su particular percepción de la probabilidad de error, sería agregada por la Sociedad Abierta en una revaluación de la probabilidad de error que no tiene por qué ser cada vez menor.

\section{Sustrato social de las instituciones, cultura y lenguaje}

El análisis efectuado en el epigrafe 5 en relación a un Banco Central independiente permite generalizar conjeturando que toda institución macrocconómica de diseño es precaria. Tres comentarios se imponen. Primero, esta precariedad, cuando se analiza en tiempo virtual, tiene que reflejarse en la fragilidad del equilibrio dinámico que corresponde a la eliminación del sesgo inflacionario. Segundo, el cambio de régimen que supone la introducción de una nueva institución no pucde analizarse en tiempo virtual y requicre un modelo en tiempo real. Tercero, la causa última de la fragilidad que estamos destacando está en la racionalidad: ésta es como un cuchillo afilado que acaba hiriendo de muerte cualquier arreglo destinado a paliar su poder letal.

${ }_{22}$ A este ensanchamiento de la noción de racionalidad se refiere la intención de J. F. Ályarez de singularizar la conducta inteligente como algo que puede ser más Racional que la conducta racional, Ver J. F. Alvarez, «¿Es inteligente ser racional?, Sistema, 109, 1992.

23 Naturalmente, la idea de que el sistema de precios agrega la información se debe a Hayek; pero la elaboración filosófica de esta idea más allá del propio mercado y en favor de una sociedad tolerante y liberal pucde seguirse, por ejemplo, en los trabajos de John Gray citados en la nota 5. 
De estos comentarios se sigue que debemos explorar la estabilidad de las instituciones macroeconómicas considerándolas como sostenidas por el equilibrio de un juego dinámico en tiempo real en el que quepa preguntarse por el enraizamiento de ese equilibrio en las convenciones sociales y en donde la introducción de limites a la racionalidad permita mantener una reputación, modelada ahora como una estrategia que se va configurando en el tiempo. Hay muchas formas de modelar un juego así; pero aquí, en donde sólo se pretende sugerir algunas ideas, es conveniente utilizar la forma evolutiva ${ }^{24}$. Supongamos, pues, dos poblaciones, una de Sindicatos y otra de Bancos Centrales. La interpretación de este supuesto es delicada en este contexto. La más sencilla es que uno y otro agente pueden utilizar estratcgias mixtas, concebidas como diferentes distribuciones de probabilidades sobre el soporte $(=$, + ). En cada momento de tiempo un Sindicato y un Banco Central son emparejados para jugar cl jucgo estático representado en el gráfico 5 sin información completa y, a la vista del resultado obtenido, modifican cl conjunto de estrategias mixtas disponibles. Esto genera una dinámica de las estrategias que supondremos exhibe cierta irracionalidad reflejada en el hecho de que la dinámica es miope (no va directamente a la única solución de Nash del juego estático) y tambićn incrcial (no toda estrategia mixta jugada y mostrada inferior a otra es eliminada inmediatamente). En estas condiciones, una dinámica así puede tener uno o varios equilibrios estacionarios localmente estables (que no tienen por qué colapsar en algún equilibrio de Nash estático) ${ }^{25}$. Si los tiene, cada uno de ellos constituye una convención social, según la definición de David Lewis, es decir, una pauta de conducta que los agentes siguen, que esperan que los otros sigan y que cada uno quiere seguir si los demás la siguen ${ }^{26}$.

La lección a aprender de este análisis informal es que es posible que el sesgo inflacionario nulo sea una convención social. Si llega o no a serlo depende tanto de las condiciones iniciales como de las particularidades de la dinámica de las estratcgias mixtas que prcvalezcan en una sociedad determinada. No

"El trabajo de A. Matsui especialmente redactado para el númcro 60 de Cuadentos Económicos de ICE, «La Teoría de Jucgos Evolutivos: desarrollo teórico y algunas aplicaciones», 1995, constituye una magnifica panorámica de modelos dinámicos en tiempo real. Los modelos cvolutiwos parecen especialmente útiles para estudiar las instituciones, entre otras cosas porque permiten preguntarse por el cambio de instituciones. A esto se hará una alusión en el comentario final de este trabajo. En cualquier caso, la interpretación de estos modelos no es estrictamente «biológica».

${ }^{3}$ Estos equilibrios son, en general, un subconjunto de los equilibrios de Nash estáticos tal como muestra D. Friedman en «Evolutionary Games in Economics», Econontetrica, 59, 1991. Por esta razón, la Teoría de los Juegos Evolutivos se suele entender como una contribución al problema de la selección entre equilibrios de Nash. El artículo de Friedman, en el contexto de estrategias puras, implicaría que el único equilibrio a considerar seria el único equilibrio de Nash existente, el que sosticne cl sesgo inflacionario. Pero esto no tiene por qué ser cierto si consideramos estrategias mixtas, ya que entonces pueder haber, en general, varios equilibrios de Nash.

23. Ver el libro de D. Lewis Convention: A Philosophical Study, Harvard University Press, 1969. 
hay, pues, ninguna garantía de que las convenciones sociales sostengan un óptimo. Sin embargo si, de hecho, lo sostienen en el sentido de que existe una convención social según la cual el Banco Central (así como el Sindicato) juega casi siempre = (aviso exigido por la naturaleza mixta de las estrategias), diríamos que ese Banco Central no es una institución de diseño, sino una institución enraizada en las costumbres de una sociedad determinada $y$, en consecuencia, afirmariamos que no adolece de ninguna fragilidad ${ }^{27}$. Todo $\sin$ relación alguna a la independencia del Banco Central.

Para cerrar la estrategia intelectual de este ensayo pensemos que, cuando nos movemos en el contexto de estos juegos en tiempo real, los experimentos intelectuales deben estar condicionados por la historia, es decir, debemos empezar el análisis desde una situación inicial dada. Supongamos, pues, que estamos en la casilla $D$ de la matriz de pagos del gráfico 1 que exhibe el sesgo inflacionario. Lo que realmente hemos visto en tiempo virtual, a saber, que la casilla $A$ podría ser un equilibrio con reputación, no se aplica si postulamos que inicialmente estamos en D, ya que no hay manera de salirnos de ahí aunque sî sea cierto que si nos saliéramos podríamos obtener la solución A como un equilibrio dinámico con reputación. Lo que nos falta por ver ahora es cómo en tiempo real se puede pasar de $\mathrm{D}$ a $\mathrm{A}$, si es que se puede ${ }^{28}$.

La forma en que vamos a hacerlo nos permite además entender específicamente un mecanismo de reputación y puntualizar la exigencia, ya destacada en repetidas ocasiones, de un lenguaje entendible por los agentes relacionados con la institución de que se trate. Para ello, volvamos a la descripción en tiempo real de un juego evolutivo que acabamos de efectuar y especifiquemos ahora que la dinámica de las estrategias corresponde a la dinámica de la mejor respuesta. Además se supondrá que, en cada momento de tiempo y antes de jugar el juego del grăfico 1 o del gráfico 5, cabe la posibilidad de cheap-talk

${ }^{27}$ Hay una forma alternativa y adicional de ver cómo la estabilidad de las institucioncs macroeconómicas dependen de la cultura. Recordemos la sugerencia de Auman estudiada en el epígrafe 6. $Y$ ahora pensemos que el conocimiento mutuo de racionalidad ajena puede ser también alcanzado como una convención social resultado de una dinámica especifica. Pues bien, se podría razonar de la siguiente manera hipotética pero que no deja de tener algún realismo. En Alemania la racionalidad ajena es conocimiento común y, en consecuencia, el óptimo $(=,=)$ no es una solución del juego representado en el gráfico 1. En Gran Bretaña, por el contrario, hay una cierta convención social que exige hacer como si no se supiera de forma común que los otros son racionales $y$, en consecuencia, el óptimo $(=,=)$ puede ser la solución del juego del gráfico 1. Diriamos que la cultura inglesa no exige un Banco Central Independiente aunque, por otro lado, introducirlo no representa ningún drama, pues nada cambia. Diriamos similarmente que la cultura alemana exige un Banco Central Independiente y que, en consecuencia, cuando hay amenazas en el horizonte (como es el caso de la sustitución del Bundesbank por el Banco Central Europeo) la resistencia es bastante notable.

${ }^{2 *}$ Lo que sigue es una adaptación al presente contexto del trabajo de A. Matsui «Cheap-talk and Cooperation in Society", Journal of Economic Theory, 54, 1991. Notemos que en este trabajo la matriz de pagos conforma un verdadero dilema del prisionero y que es necesario entonces utilizar estrategias condicionadas en los anuncios y/o los comportamientos ajenos. En nuestro caso esto no es necesario. 
de forma que cada jugador puede anunciar si jugará $=0+$. Por tanto, las estrategias son estrategias dobles del tipo «anuncio $X$, hago $X$ '» en donde $\mathrm{X}$ puede tomar los valores $=\mathrm{o}+\mathrm{y}$ en donde $\mathrm{X}^{\prime}$ es una función más o menos compleja de $(=,+)$. Tal como veremos, la reputación puede ser muy fácilmente modelada como la credibilidad de los anuncios que se emiten en el cheap-talk. En este contexto el punto de partida es «anuncio +, hago +» por parte tanto del Gobierno como del Sindicato.

GRAFICO 1

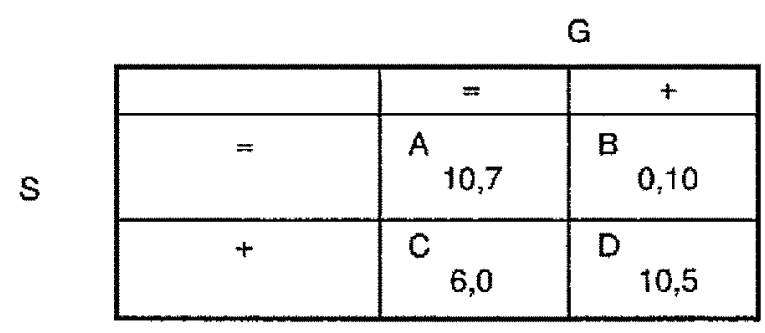

Comencemos el análisis por el juego que correspondería a la matriz de pagos de un Gobierno débil (según el gráfico 1 que se reproduce aquí por conveniencia). Es muy fácil ver que este gobierno débil sólo puede adquirir reputación de débil y que, de hecho, actuará como débil. En efecto, coloquémonos en el punto inicial en el que el Gobierno anuncia + y hace + . Anunciar ahora = no es creíble por parte del Gobierno, ya que hacer + es estrategia dominante. Sólo queda, pues, anunciar + , que sí que es un anuncio creíble. ¿Cabe ahora que $\mathrm{G}$ haga realmente $=$ ? La respuesta es que no. Si el Sindicato creyera el anuncio del Gobicrno de que va a hacer + , cste Sindicato haria también + en lugar de $=(10$ contra 0$)$, con lo que el Gobierno preferiría hacer + y no $=(5$ contra 0$)$. Si el Sindicato no creyera el anuncio del Gobierno de que va a hacer + , y creyera, por tanto, que va a hacer $=$, este Sindicato haría también $=y$ no $+(10$ contra 6$)$ con lo que el Gobierno preferiría hacer + (10 contra 7). Luego el Gobierno $G$ siempre jugará la estrategia doble «anuncio $t$, hago + . Como en el equilibrio, siempre que anuncia + hace + , un lenguaje ha sido creado: decir «voy a hacer $+\gg$ significa que hace + . Además, el anuncio es perfectamente creible y el Gobierno adquiere reputación de débil. La clave técnica de este resultado es que «anuncio $=$, hago $=»$ no es la mejor respuesta a la situación inicial «anuncio + , hago $+»$. De hecho, la única mejor respuesta a esa situación inicial es ella misma, sin duda mejor que la estrategia mentirosa que acabamos de examinar.

Cabe, pues, preguntarse si no sería conveniente establecer un Banco Central independiente con las preferencias que configuran el gráfico 5 (que se reproduce aquí por conveniencia). 
GRÁFICO 5

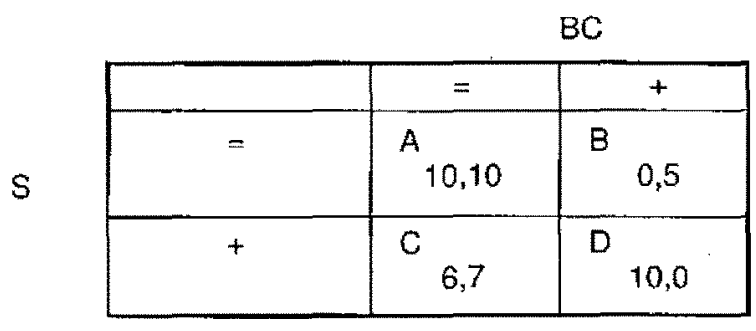

¿Puede ahora este Banco Central pasar de la casilla $D$ a la casilla $A$ ? La respuesta ahora es que sí, ya que «anuncio $=y$ hago $=»$ es la mejor respuesta a la situación inicial heredada por el Banco Central. Véamoslo. Si el Banco Contral anuncia que va a hacer $=$, el Sindicato puee creerlo o no. Si el Sindicato cree en el anuncio él también jugará = y hemos acabado ya que, en ese caso, al Banco Central le convicne cumplir con lo anunciado. Si el Sindicato no cree en el anuncio del Banco Central y, por tanto, cree que este jugará + , él, el Sindicato, jugará $+(10$ contra 0$)$, con lo que ahora al Banco Central le interesa seguir jugando $=(7$ contra 0$)$. El lector puede verificar que las estrategias mentirosas son peores respuestas a la situación inicial que la del «anuncio $=$, hago $=»$, con lo que esta estrategia es la mejor respuesta. El Banco Central, pues, anunciará = y hará =, con lo que eventualmente el Sindicato acabará jugando (y anunciando) $=$. Una vez más se ha crcado un lenguaje: «voy a hacer $=$ significa que hago = y la reputación está adquirida en el sentido de que se creen los anuncios.

Es de esperar que, a pesar de que el presente contexto no corresponde exactamente al de Matsui (en cuanto que la matriz del gráfico 1 no es un dilema del prisionero para ambos, y sobre todo en cuanto que aquí cada población sólo puede entenderse como un conjunto de estrategias mixtas diferente), su análisis, correspondiente a su trabajo citado en la nota anterior, pudiera aplicarse de forma que, de facto, la dinámica de la mejor respuesta lleve en tiempo real a la situación A como elemento de una noción de equilibrio denominada Social Stable Set y elaborado por él mismo y Gilboa ${ }^{20}$. Suponiendo que así fuera, podríamos dccir que el Banco Central está cnraizado en las costumbres del país de que se trate y que, además, ahora se relaciona con otros agentes cn un lenguaje inequívoco en el que expresa sus intereses y en el que es entendido y creído dada su reputación de veraz.

Para finalizar este epígrafe cabe todavía un comentario tangencial con la ética y con la elaboración de lenguaje. Notemos que en una posible ética de la población de Sindicatos la idea de jugar una estrategia dominada (poner

* Ver I. Gilboa y A. Matsui, "Social Stability and Equilibrium", Economerrica, 59, 1991. 
la otra mejilla en lugar de salir corriendo) pudiera ser completamente aberrante. Podría serlo, dentro del ejemplo que estamos manejando, porque ninguna de las dos posibles estrategias domina a la otra (por eso el gráfico 1 no corresponde al dilema del prisionero normal) y, porque, en consecucncia, nunca se ha jugado. Análogamente, dentro de la población de Bancos Centrales esa práctica no es aberrante. Un problema de la ética, de cualquier ética, es que quizá no cs universal. ¿Nos lleva esto al relativismo moral? Lo interesante es que, según el ejemplo del texto, cabe la elaboración de un lenguaje en el que las dos poblaciones pueden comunicarse y convivir e incluso alcanzar un óptimo sin necesidad de convertirse mutuamente. Así es como un vulgar economista entendería las propuestas éticas de Habermas basadas en su teoría de la acción comunicativa ${ }^{30}$.

\section{Conclusión y comentarios finales}

En este trabajo he intentado contribuir someramente a lo que se podría denominar la Economia Institucional. Más en concreto he procurado dilucidar el papel y la precariedad de un cierto tipo de instituciones, aquellas que involucran el comportamiento de un agente que refleja la soberanía estatal. Si cste aspecto de la Economía Institucional tiene algún interćs cspecifico es porque dicho agente está, por definición, por encima de los agentes privados, y es el único que puede permitirse no cumplir los acuerdos. La aportación de cste trabajo, si es que existe, puede resumirse muy fácilmente. Es posible que la introducción de estas instituciones macroeconómicas sea algo precario y hasta peligroso a no ser que estén sostenidas por convenciones sociales, en cuyo caso quizá no sostengan un óptimo $y$, desde luego, no tienen por qué ser comunes a todas las sociedades, ya que las convenciones sociales, en cuanto representan equilibrios relativos a una dinámica determinada y a unas condiciones iniciales dadas, pueden no corresponder a un óptimo que no tuviera en cuenta estas rcstricciones y difícilmente serán comunes a todas las sociedades.

Además, y como el argumento ha seguido el hilo conductor de la problemática de la independencia de un Banco Central, este tema ya clásico también ha sido problematizado recogiendo ideas ya anteriormente publicadas ${ }^{31}$. Los argumentos empíricos que se suelen esgrimir para predicar la suficiencia de la independencia del Banco Central a efectos de desinflar la economía no

${ }^{30} \mathrm{Mi}$ ignorancia de estos temas estă, sin embargo, paliada por la lectura de Conciencia moral y acción comunicativa de J. Habermas, Península, 2." ed., 1991, especialmente los ensayos II y III, y del ensayo de J. Muguerza «De la conciencia al discurso: ¿un viaje de ida y vuelta?», en La Filosofia Moral y Politica de Jürgen Habermas, J. A. Gimbernat (ed.), Biblioteca Nueva, 1997.

31 Además de los comentarios referenciados en las notas 3 y 8 , gran parte de lo expuesto aquí está en los siguientes trabajos: "Historia y Diversidad. Un ensayo de Filosofía Económica», Ekonomiaz, 35, 1996, y «Una sugerencia para plantcarse cl problema de la reputación del Banco 
son convincentes. Esta opinión queda reforzada si además nos percatamos de que, en abstracto, un Banco Central, aun siendo independiente, quizá no pueda alcanzar la reputación necesaria, quizá la pierda de repente si llega a alcanzarla y quizá imponga a la economía unos costes muy grandes en su intento por alcanzarla. Sin embargo es posible que todo esto no ocurra si el Banco Central de un país concreto está enraizado en las costumbres de dicho país. En ese caso, y bajo ciertas condiciones, el Banco Central puede ganarse una reputación mediante unas actuaciones que correspondan a unos anuncios emitidos en un lenguaje propio. No parece, sin embargo, que la independencia tenga mucho que ver con todo esto.

A lo largo de la exposición ha sido posible examinar algunos temas de raigambre filosófica y enfocarlos con óptica de economista. La cuestión de las metapreferencias es un asunto sutil y para el que no parece existir ninguna solución satisfactoria. La única forma razonable de entenderlo para un economista de hoy es no limitarse a aspectos formales de coherencia interna, sino aplicar nociones de juegos estratégicos entre los sujetos que quedan después de suponer la escisión de la personalidad. Respecto al tema genérico de la racionalidad, lo que se desprende de este ensayo es que quizá podría extenderse la noción de racionalidad hasta abarcar algunas conductas no egoistas observadas pero aparentemente irracionales, conformando así una noción de Racionalidad con mayúscula. Estas formas no egoístas de actuar podrían considerarse imprudentes o inteligentes dependiendo del contexto; pero no necesariamente irracionales. En cuanto a la posible defensa de una sociedad abierta basada en la falibilidad del conocimiento, parece que resultaría problematizada si admitiéramos que la racionalidad ajena es sólo conocimiento mutuo de orden $\mathrm{N}$ y no conocimiento común porque, en ese caso, la libre interacción no garantiza, parece ser, la conservación del conocimiento acumulado. Asimismo hemos sido capaces de perfilar una sugerencia que permite a un economista tratar de entender la comunicación entre dos éticas distintas, correspondientes a sociedades distintas, como un problema simultáneo de creación de lenguaje y de determinación de la acción en el contexto de un juego dinámico jugado por las poblaciones correspondientes.

Finalmente hay que referirse a un tema subyacente a todo el ensayo y que está en la base de posibles desarrollos ulteriores de las ideas aquí expresadas. Se trata de la tensión entre la universalidad exigida por la racionalidad y la potencialidad de un "toque de irracionalidad" que redunda, sin embargo, en diversidad. La racionalidad total, que presumiblemente nos llevaría a un óptimo, implica sin embargo la fragilidad de algunas instituciones, las que hemos llamado macroeconómicas. Para que éstas fueran menos precarias deberían estar entroncadas en las costumbres del país y desarrolladas a través de un proceso en el que la racionalidad es limitada. Pero en ese caso podría pensarse que cada

Central Europeo», Perspectivas del Sistena Financiero, 58, 1997, de los que me he permitido traer aquí ocasionalmente pasajes enteros. 
sociedad, con condiciones iniciales dadas y con cultura diferenciada, tendría sus instituciones propias ninguna de las cuales tiene en principio ninguna presunción de optimalidad. El contraste entre dos pares, homogeneidad/optimalidad por un lado y diversidad/suboptimalidad por otro, puede ser muy rico en implicaciones. Pensemos, por cjemplo, en una analogía biológica que podríamos utilizar en favor de la diversidad. Los seres humanos como agentes sociales actuarían como se dice que actúan los genes, es decir, constituyendo diferentes vehículos en el caso biológico, o instituciones diversas en el caso económico, cada uno de los cuales se organiza y organiza a su entorno de forma alternativa.

De esta forma se maximiza la capacidad de sobrevivir cuando alguna catástrofe ocurre. Los economistas, sin necesidad de analogías bilológicas, saben bien que la perfección es frágil. Por ejemplo, los repartos de uno u otro tipo suelen ser del tipo «proporcional» que rara vez es el más adecuado para cualquier criterio; los contratos de muy distinta índole que se observan en áreas muy diversas suelen ser «simples» cuando cn cada caso hay un contrato «complejo» óptimo. Lo que la moraleja de este trabajo quiere resaltar es precisamente esto, que la proliferación de instituciones alternativas y subóptimas puede ser una forma «inteligente» de confrontar los avatares de la vida social cuando cada una de ellas es el resultado de un proceso dinámico con un «toque de irracionalidad».

Esta forma de defender la diversidad pudiera resultar sospechosa para quienes ven en el tradicionalismo ciego una inhibición política que puede alcanzar incluso el dogmatismo del "pensamiento único», o para quienes sospechan que el relativismo moral no es sino una forma de quitarse de encima la responsabilidad de atajar mucha crueldad innecesaria. Sin embargo, el marco de análisis aquí esbozado es adecuado para estudiar precisamente estos problemas. Aunque hacerlo tendrá que esperar a otra ocasión, las líneas generales de ataque son inmediatas. Las ideas pragmatistas de Rorty respecto a la inevitable preminencia del «nosotros» a efectos del relativismo moral pueden completarse con el análisis dinámico en tiempo real aquí mencionado cuando dos o más «nosotros» distintos se funden en uno solo. Es de esperar que, aunque en ocasiones un «nosotros» se coma al otro, quede abierta la posibilidad de que surjan convenciones sociales o reglas éticas novedosas, y quizá superiores en algún sentido, a las existentes en aislamiento. En cuanto a la inhibición política, se puede decir que, lejos de sostenerla, el marco de ideas aquí manejado dota de sentido a la actividad política y a la experimentación social. Lo primero, porque las convenciones sociales pueden no ser equilibrios evolucionariamente estables (es decir, a prueba de "mutantes») y es precisamente entonces cuando ser un «mutante» merece la pena, pues puede llevar a cambiar la convención social, y, por tanto, a la sociedad, mediante la persuasión. De becho, la proliferación de mutantes o, lo que es lo mismo, el ejercicio continuado de la política, es el único procedimiento que tenemos para testar la fortaleza de nuestras instituciones, una conclusión muy diferente de la que alcanzan los 
que piensan que enfrentarse al «pensamiento único» es necedad. Lo segundo, la experimentación social, cobra sentido porque se trata de un expediente que convierte en estocásticos los comportamientos de los agentes y porque esta estocasticidad consigue aislar un único de los equilibrios posibles a pesar de las diversas condiciones iniciales. Persuasión y experimentación recuperan ahora una funcionalidad social que no pueden sonar tener cuando las sociedades pretenden guiarse por ideas hiperracionales y desnudas de toda adherencia histórica. Pero todo esto comienza a ser otra historia ${ }^{32}$.

${ }^{32}$ Durante la redacción de este ensayo he tenido la oportunidad de leer el artículo de $\mathrm{J}$. A. Rivera en el número 78 de Claves de Razón Práctica, correspondiente a diciembre de 1997 y titulado «La hipótesis de la Reina Roja y otras hipótesis». Ambos trabajos, el de Rivera y el presente, tiencn en común el interés por las instituciones consideradas como equilibrio de procesos dinámicos en tiempo real. Sin embargo, el trabajo de Rivcra está mucho más cerca de una concepción biologista de estos proccsos y mucho menos in teresado en la posible precariedad de las instituciones macroeconómicas. A pesar de ello, el hecho de que preste atención a algunos problemas keynesianos de hacienda pública mucstra que quizá el esfuerzo de tender puentes entre filốsofos y economistas y otros científicos sociales quizá encuentre eco. 